\title{
Development of the Holifield Radioactive Ion Beam Facility
}

\author{
B.A. Tatum for the HRIBF Operations Staff \\ Oak Ridge National Laboratory*
}

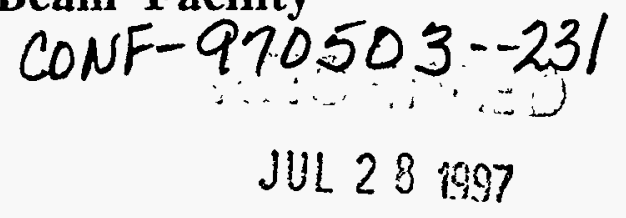

\section{Abstract}

The Holifield Radioactive Ion Beam Facility (HRIBF) construction project has been completed and the first radioactive ion beam has been successfully accelerated. The project, which began in 1992, has involved numerous facility modifications. The Oak Ridge Isochronous Cyclotron has been converted from an energy booster for heavy ion beams to a light ion accelerator with internal ion source. A target-ion source and mass analysis system have been commissioned as key components of the facility's radioactive ion beam injector to the $25 \mathrm{MV}$ tandem electrostatic accelerator. Beam transport lines have been completed, and new diagnostics for very low intensity beams have been developed. Work continues on a unified control system. Development of research quality radioactive beams for the nuclear structure and nuclear astrophysics communities continues. This paper details facility development to date.

\section{INTRODUCTION}

The Holifield Radioactive Ion Beam Facility (HRIBF) [1] of Oak Ridge National Laboratory was formally dedicated on December 12,1996, and approved for high intensity operation as a National User Facility, the first of its kind in North America. The HRIBF will be the first radioactive ion beam (RIB) facility in the United States devoted to low-energy nuclear structure and astrophysics research. Light ion beams from the Oak Ridge Isochronous Cyclotron (ORIC) are utilized to bombard targets in the target/ion source (TIS) assembly on the $-300 \mathrm{kV}$ RIB injector platform. Radioactive ions diffuse from the target and are ionized, then mass separated. Subsequently, the RIB is transported to, and accelerated by, the $25 \mathrm{MV}$ tandem electrostatic accelerator (Tandem) prior to transport to any of several experimental devices.

\section{ORIC DEVELOPMENT}

ORIC development centers around the production of proton, deuteron, and alpha beams which are required for initial RIB production. The operational objectives are to increase beam energy and intensity while minimizing machine activation, and also to increase machine reliability. Goals and present status of beam development are shown in Table 1. Beam intensities Q intentionally limited by source aperture size during the development phase to minimize machine activation. The ORIC has provided up to about 5 microamps of $42 \mathrm{MeV}$ protons to the target/ion source to produce ${ }^{69} \mathrm{As}$ and ${ }^{70} \mathrm{As}$ beams. The beam current is being increased in stages to study radiation and beam-heating effects on the target materials and to minimize residual radioactivity. Thus far, no adverse effects have been observed. Proton beam current will be increased to $50 \mu \mathrm{A}$ as development progresses to produce RIBs of sufficient intensity for the experimental research program.

Table 1: ORIC Beam Development

\begin{tabular}{|c|c|c||}
\hline Beam & Goal & Status \\
\hline proton & $60 \mathrm{MeV}, 50 \mu \mathrm{A}$ & $42 \mathrm{MeV}, 5.0 \mu \mathrm{A}$ \\
deuteron & $50 \mathrm{MeV}, 50 \mu \mathrm{A}$ & $49 \mathrm{MeV}, 0.4 \mu \mathrm{A}$ \\
alpha & $75 \mathrm{MeV}, 200 \mu \mathrm{A}$ & $75 \mathrm{MeV}, 1.6 \mu \mathrm{A}$ \\
\hline
\end{tabular}

ORIC is in the middle of a four year Accelerator Improvement Project (AIP) to accomplish the stated objectives. Central region development for separated turns and high extraction efficiency is a focal activity. Presently, extraction efficiencies are less than $50 \%$, but modifications currently underway are designed to increase efficiencies to $75 \%$. The result will be improved beam production with less activation of key components such as the electrostatic deflector and extraction channels. New ion source heads and an improved gas handling system have resulted in cathode lifetimes in excess of 200 hours. The design simplifies source maintenance for minimal personnel radiation exposure. Improved beam diagnostics have been installed including a multi-segmented radial probe and beam scanning system which provides both radial and axial beam profiles.

Machine reliability has been greatly increased in several areas. The deionized water system has been simplified and flow switches rebuilt for reliable component protection. Several aging power supplies have been or will be replaced, eliminating chronic down-time. A majority of ORIC controls have been moved to the Vista control system from the obsolete Modcomp and older hardwired systems. Complete controls wiring and interface module replacement has resulted in a more robust and easily maintainable system. RF system modifications are also a

\footnotetext{
- Research sponsored the U.S. Department of Energy under contract number DE-AC05-960R22464 with Lockheed Martin Energy Research Corporation,.

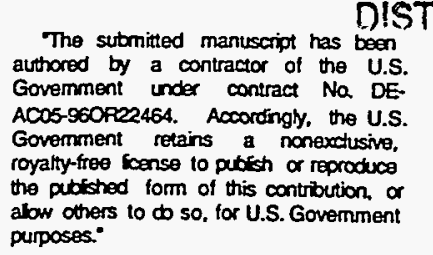
DISTRIBUTION OF THIS DOCLMENT IS UNLIMITED

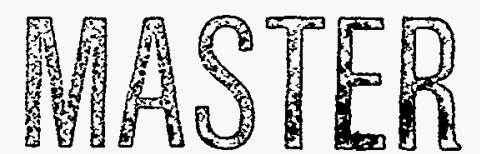




\section{DISCLAmIER}

Portions of this docament may be illegible in electronic image products. Images are produced from the best available original document. 
part of the AIP Project, including the replacement of the $20 \mathrm{kV}, 30 \mathrm{~A}$ power supply required by the anode of the RCA 4648A power amplifier tube.

\section{RIB INJECTOR DEVELOPMENT}

The RIB injector [2] consists of all equipment on the $-300 \mathrm{kV}$ platform (target ion/source, first stage magnetic mass separator, electrostatic quadrupoles, and cesium vapor charge exchange cell) and the transfer beam line (electrostatic quadrupoles and second stage magnetic mass separator) to the $25 \mathrm{MV}$ Tandern. Great progress has been made in understanding and optimizing the performance of the elements of the RIB injector.

Routinely, the beam exiting the ion source via the extract electrode is steered onto the optical axis of the RIB injector by using the first two of the four electrostatic quadrupoles immediately after the ion source as steerers. The first steerer bends the beam so that it crosses the optical axis at the position of the second steerer. The second steerer bends the beam parallel to the optical axis.

Total transmission through the RIB injector from the ion source to the Tandem is $28 \%$ for ${ }^{71} \mathrm{Ge}$ ions. Essentially all loss is in the charge exchange cell; transmission of positive ions through the cell with no cesium vapor is $70 \%$, and the measured negative ion fraction is $40 \%$. All other elements, including the second stage mass separator, transmit the beam without measurable loss. The transmission of the entire system from the ion source through the Tandem is about $6 \%$, corresponding to a transmission through the Tandem itself of $20 \%$. These measurements were made with a $50 \mathrm{keV}{ }^{70} \mathrm{Ge}$ beam on the high voltage platform, accelerated to $200 \mathrm{keV}$, and injected into the Tandem.

The design mass resolution of the second stage mass separator is 1 part in 20,000 and is limited by the size of the beam at the object of the separator in the dimension corresponding to the bending plane of the separator. Transmission of $100 \%$ has been achieved with a size corresponding to a resolution of 1 part in 7500 (FWHM). With this tune, a resolution of 1 part in 10,000 with $60 \%$ transmission or 1 part in 20,000 with $20 \%$ transmission is achievable. Based on analysis of an experimental study of the energy loss straggling in the charge exchange cell, the intrinsic resolution of the second stage mass separator is conservatively estimated to be better than 1 part in 10,000 . Operation of the second stage mass separator with full transmission at the design mass resolution will be the major development project on the RIB injector during the coming year.

After bombardment with an intense light ion beam, the TIS vacuum enclosure may contain up to $100 \mathrm{Ci}$ of residual activation, prohibiting prompt manual removal of the TIS from the $-300 \mathrm{kV}$ platform for examination, repair, or interchange. Thus, to minimize delays in the experimental program, a Remote Handling System (RHS) is used to remove the TIS from the platform.

The RHS consists of three major components: (1) a sixdegree of freedom robotic arm, designed to withstand $10^{8}$ RAD total dose, mounted on the high voltage platform next to the TIS; (2) a conveyor that extends from the high voltage platform room through the cyclotron vault and into a shielded storage room; and, (3) a robotic gantry crane in the shielded storage room.

The RHS only operates with ORIC beam off, and platform and TIS high voltage off. The TIS is remotely disengaged from the RIB injector vacuum system and moved to a retracted fiducial position on the TIS table. The robotic arm picks up the TIS, piaces it in a stainless steel Contamination Control Box (CCB) which sits on the remotely-extended end of the conveyer, and places a dust-tight cover on the CCB. The end of the conveyer with the $\mathrm{CCB}$ retracts into the wall and the conveyor moves the CCB to the shielded storage room where it is picked up by the robotic gantry crane and placed inside a lead cask. The crane is capable of placing a lead lid on top of the cask. The RHS also reverses this sequence to install a new TIS on the RIB injector. RHS reliability was confirmed by the successful performance of 50 consecutive repetitions of the removal and installation processes using a 250 pound source mock-up, which is twice the weight of a normal TIS. The time required to remove and install a source was 41 minutes, and no human intervention was required during the 34 hour period. The RHS is fully programmable and communicates with the RIB injector VISTA-based control system, and also has manual controls.

Several engineering modifications have been made to the RIB Injector. All air and water valves associated with the TIS have been moved to the instrumentation platform. The extract electrode drive mechanism has been moved to the top of the Faraday cage to minimize radiation damage to the drive motor and associated limit switches. Flexible spring mountings have been installed on the extract electrode to allow easier movement. The self sealing features of the water connections to the TIS have been removed to improve reliability of cooling water flow. The positioning pin arrangement for the TIS base plate and table has been modified to accommodate the RHS. All elastomer $\mathrm{O}$-rings in the extraction region have been replaced with metal O-rings. The acrylic supports for the deceleration tube and for the extraction area ceramic insulators have both been replaced with radiation resistant G10 supports. 


\section{TANDEM ACCELERATOR DEVELOPMENT}

The Tandem is fully devoted to RIB development, routinely providing stable ion beams in support of TIS studies and experimental apparatus commissioning during periods between RIB acceleration.

The combination of the Tandem and the on-line isotope separator (UNISOR) provides a valuable tool for testing new target materials and ion source concepts. The high reliability and low operating cost of the Tandem has permitted completion of 15 experimental runs over the past year for target/ion source development. Thus far, the Tandem has provided both proton and deuteron beams for this program and addition of a helium negative ion source is being considered. The proton beam is administratively limited to an energy of $40 \mathrm{MeV}$ and current of $10 \mathrm{nA}$ due to shielding limitations around the UNISOR target region and the desire to limit activation of the Tandem components. The high sensitivity of the isotope separator allows measurements to be carried out at these low beam intensities while minimizing residual radioactivity and personnel exposure to radiation. The emphasis during the past year has been on development of liquid germanium targets for production of ${ }^{69} \mathrm{As}$ and ${ }^{70} \mathrm{As}$ beams, and alumina targets for production of ${ }^{17} \mathrm{~F}$ and ${ }^{18} \mathrm{~F}$ beams. The first program is essentially completed and an ${ }^{69} \mathrm{As}$ beam has been provided to the experiment end station. The fluorine beam development program has had good success using thin aluminum oxide fiber targets and a negative ion source is being developed to provide these beams to the research program in the near future.

Tandem equipment has been upgraded for improved safety, environmental compliance and better operation for the RIB experimental program. For improved safety, the Tandem radiation safety system was upgraded for three reasons: (1) to comply with DOE order 5480.25 and related ORNL Facility Safety Procedure, (2) to improve system electrometers by replacing old electrometer circuitry which was based on obsolete electronics and (3) to extend the system to the new Recoil Mass Separator (RMS) experimental area to provide the convenience of a permissive entry system to RMS experimenters.

To comply with freon-reduction guidelines, the Tandem stable-ion injector's Freon 113 cooling system was replaced with a recirculating water-cooled system with a liquid-to-air heat exchanger. All components of the system are installed in the limited space on the ion source platform. A mixed-bed resin deionizer keeps water conductivity at 14 megaohm-cm.

Tandem energy-analyzing magnet coils were replaced with new coils of a modified design in August 1995 after instabilities became apparent during a period of routine accelerator operation in February of 1994. The cause of instabilities was an internal short in one of the magnet coils. The magnet was operated with fewer turns for several months at a 50\% reduction in bending power. Energy-analysis versus magnet field was calibrated using a time-of-flight method resulting in an analyzed beam energy accurate to at least $0.1 \%$.

\section{LOW INTENSITY BEAM DIAGNOSTICS}

Beam diagnostic requirements at the HRIBF vary from the measurement of high intensity ORIC beams used in production to typically low-intensity radioactive beams. Below is a list of methods and devices used for monitoring radioactive beams.

\subsection{Non-Invasive Sampling}

- An ionization sampler of the residual gas by the beam particles which is 3-D sensitive $\left(10^{11}-10^{7} \mathrm{p} / \mathrm{s}\right)$

\subsection{Invasive Readout}

- (Alumina) with sensitive CCD video camera (104$109 \mathrm{p} / \mathrm{s}$ )

- Phosphor $X-Y$ position sensitive avalanche type gas detector $(100-106 \mathrm{p} / \mathrm{s})$

- Sensor based on detecting ions or electrons emitted by the beam hitting a metal plate $\left(10^{11}-10^{7} \mathrm{p} / \mathrm{s}\right)$

The sensor, based on detecting emissions from a metal plate [3], shown in Fig. 1, is being used at several dead sections of the accelerating tubes. It is mounted with mechanical actuators that place it in the beam path.

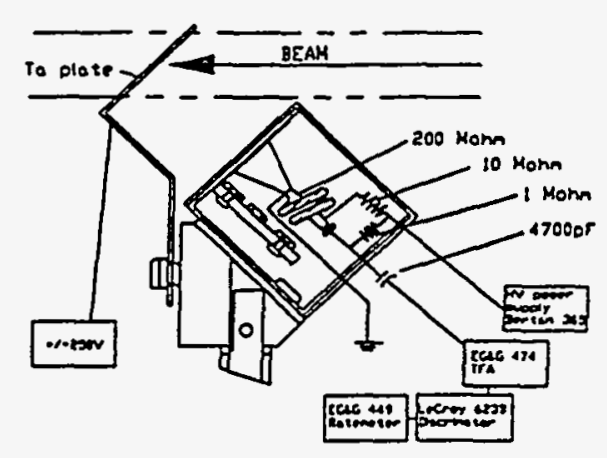

Figure 1. Invasive Readout.

\section{ACCELERATOR CONTROL SYSTEM}

The accelerator control system is built around Vsystem, a commercial toolkit from Vista Control Systems. 
Vsystem provides a foundation for a distributed, data driven system in which a real-time database is used to dynamically configure system behavior and provide a common mechanism for interprocess communication. In addition, Vsystem includes Vdraw which is an X Window based GUI builder. All human interface screens have been created with the assistance of Vdraw.

A portion of the software runs in an environment in which a VAX 4000 model 90 running VMS supports the $\mathrm{X}$ Terminals on which the Vdraw screens are displayed, and three rtVAX300 processors running VAXELN perform the $\mathrm{V} / \mathrm{O}$ processing and house the real-time databases. H/O devices include numerous RS232 and RS485 serial interface modules in addition to AllenBradley remote I/O scanners and programmable logic controllers (PLCs). The ORIC, RIB injector, and a number of beamlines are controlled by these components.

The control system upgrade for the tandem accelerator and related beamlines was started some time after work on ORIC and the injector began. At the same time, a new multiplatform version of Vsystem became available. Consequently, an Alpha workstation running Digital UNIX was chosen to support the human interface displays

\section{REFERENCES}

[1] D. K. Olsen et al., Proc. Second Int Conf on Radioactive Nuclear Beams, Louvain-la-Neuve, Belgium, August 1991, ed. By TH. Delbar (Adam Hilger, Bristol, 1992), p. 131; A proposal for Physics with Exotic Beams at the Holifield Heavy Ion Research Facility, edited by J. D. Garrett and D. K. Olsen, February 1991.

[2] D. T. Dowling, et al., Status of the Radioactive Ion Beam Injector at the Holifield Radioactive Ion Beam Facility, Proceedings of the 1995 Particle Accelerator Conference and International Conference on High Energy Accelerators, Dallas, TX, May 1-5, 1995.

[3] M. Meigs, D.Haynes, C.Jones, C.Lecroy, "A New Beam Intensity Monitoring System with Wide Dynamic Range for the Holifield Radioactive Ion Beam Facility," Proceedings of the 1995 Particle Accelerator Conference and International Conference on High Energy Accelerators, Dallas, TX, May 1-5, 1995. and a Heurikon $68060 \mathrm{CPU}$ running VxWorks was selected to control the I/O devices and house the real-time databases. עO hardware for the tandem consists of devices connected to a CAMAC serial highway and is not included in the upgrade.

Currently, the RIB injector subsystem is fully implemented, the ORIC is largely implemented, and integration testing is proceeding for the Tandem and associated beamlines. Future plans include the replacement of the VAX/VMS workstation with an Alpha workstation running Digital UNIX. In addition, AllenBradley ControlNet processors, with perhaps one Heurikon $68060 \mathrm{CPU}$, will replace the VAXELN CPUs.

\section{CONCLUSIONS}

Considerable progress has been made at the HRIBF in both radioactive ion beam and machine development. Future directions will focus on expansion of the array of RIB beams available for research and higher intensity $R I B$ production with the intent of maintaining a world-class research complex.

\section{DISCLAIMER}

This report was prepared as an account of work sponsored by an agency of the United States Government. Neither the United States Government nor any agency thereof, nor any of their employees, makes any warranty, express or implied, or assumes any legal liability or responsibility for the accuracy, completeness, or usefulness of any information, apparatus, product, or process disclosed, or represents that its use would not infringe privately owned rights. Reference herein to any specific commercial product, process, or service by trade name, trademark, manufacturer, or otherwise does not necessarily constitute or imply its endorsement, recommendation, or favoring by the United States Government or any agency thereof. The views and opinions of authors expressed herein do not necessarily state or reflect those of the United States Government or any agency thereof. 